\title{
Working Memory and Distraction: Performance Differences between College Students with and without ADHD
}

\author{
Tabitha W. Payne, Noah B. Z. Steege \\ Kenyon College, Gambier, USA \\ Email: paynet@kenyon.edu
}

Received May $30^{\text {th }}, 2013$; revised June $30^{\text {th }}, 2013$; accepted July 7, 2013

Copyright (C) 2013 Tabitha W. Payne, Noah B. Z. Steege. This is an open access article distributed under the Creative Commons Attribution License, which permits unrestricted use, distribution, and reproduction in any medium, provided the original work is properly cited.

\begin{abstract}
The goal of this study was to examine potential deficits in working memory capacity for college students that had a diagnosis of ADHD. College students with ADHD may be a particularly vulnerable group of individuals, given that success on academic work required focus and working memory for a variety of problem solving activities. Performance on these assessments involved controlled processing with simultaneous memory load. Both verbal and visual complex span tasks were used to assess working memory. Additionally, students were all administered with the Brown ADD scale in order to examine self-reported issues with distractibility. Results revealed that ADHD students performed significantly lower on the verbal complex span measure of working memory. No differences in reported inattentiveness were found. Findings were discussed in context of varying task demands in working memory and executive function measures.
\end{abstract}

Keywords: Adult ADHD; Working Memory

\section{Introduction}

The goal of this study was to further examine the hypothesis that working memory deficits are consistent with a diagnosis of Attention Deficit/Hyperactivity Disorder (ADHD) in young adult college students. This population is of particular interest since attaining academic success at the collegiate level requires a high degree of controlled attention and executive processing. Working memory capacity can be defined as an individual's ability to control attention for the purpose of maintaining information in awareness for the purpose of further processing (e.g., integrating information, transforming information, or rehearsal for later use). This individual capacity is important for academic success in a wide array of activities, such as reading comprehension (Daneman \& Carpenter, 1980), explicit learning (Unsworth \& Engle, 2005), verbal, spatial, and figural reasoning (Kane et al., 2004), second language processing (Payne, Kalibatseva, \& Jungers, 2009), and even note-taking and following directions (Engle, 2002). It is well-established in the literature with neurotypical samples that individual differences in working memory capacity predict performance on selective attention measures, from filtering extraneous information during the dichotic listening task (Conway, Cowan, \& Bunting, 2001) to controlling reflexive eye movements to avoid capture of attention from an orienting stimulus (Bleckley et al., 2003). Finding a connection between ADHD and working memory measures will provide concurrent validity for assessments what already been shown to be associated with individual differences in attentive processing with a non-diagnosed population.

It seems highly likely that working memory deficits would be observable with a young adult college sample with ADHD since there is evidence that both elementary school-aged children and high school students with ADHD show such per- formance decrements In a study by Kofler et al. (2010), ADHD boys between the ages of 8 and 12 were compared to typically developing control children on both verbal and visuospatial working memory assessments. The verbal measure required memory for a serial list of numbers and a letter, with a subsequent transformation of order, prior to recalling a set. The visuospatial measure involved memory for where targeted dots appeared in a series of grids. There was a significant difference in the performance of children with and without ADHD diagnosis, which became more prevalent with increased memory set size, or load on working memory. Consistent findings appear with high school students with ADHD symptoms, showing difficulty in both verbal and visual working memory recall (Caterino \& Verdi, 2012). When using a text model in which visual images appear prior to semantically related text passages, students without ADHD seem to benefit from this formatting style, whereas this method hurts the performance of the ADHD group, with the images serving as a distractor for text recall. Both of these studies with younger samples indicate that working memory functioning is problematic for those with ADHD diagnoses or symptoms. If working memory deficits are pronounced for ADHD, in general, then it is possibly a continuing condition into adulthood, which should be observable with comparative samples.

The research with young adult samples has produced inconsistent findings of a connection between ADHD and working memory. In a study using young adults (mean age of 25 years) who were clinically referred for psychological evaluation (by vocational rehabilitation counselors), no significant relationship 
was found between working memory performance and reported distractibility on the Brown ADD scale (Stearns, Dunham, McIntosh, \& Dean, 2004). The Brown scale score did predict a previously provided clinical diagnosis for 60 out of the 70 participants. It is possible that the lack of correlation between the Brown scale and performance on the working memory measures (WAIS-III and WMS-III) could be due to restricted range of the sample for the self-reported distractibility items on the Brown scale since the mean was fairly high for the sample, well above the criterion for ADHD diagnosis. It is important to consider a wider range of symptoms and executive functioning in the sample in order to observe the relationship between working memory and attentional difficulties. However, gaining understanding of ADHD can benefit from studies publishing with a wide array of samples.

In a study with an ADHD adults (mean age 21.3) and controls (mean age 22.1), Roberts, Milich, \& Fillmore (2012) examined potential decrements in ADHD performance with increasing load on working memory using the n-back task in which participants must rely on verbal rehearsal. Although there was no interaction between ADHD status and size of memory load on accuracy, there was an interesting finding in which reaction time on the n-back task was slower for ADHD individuals with lower memory loads (1-back and 2-back) the differences were not present with the largest load. Additionally, the participants with ADHD did show difficulty with response selection on a dual choice-response task, leaving the authors to emphasize that cognitive deficits associated with ADHD may include other more specific processes than working memory capacity. Regardless of whether memory load was found to be differentially processed by individuals with and without ADHD, the importance of this study is establishing a link to working memory, in general.

One suggestion for researchers has been to examine a wider array of measures relating to executive functions, since many standardized cognitive neurological assessments do not show sensitivity to ADHD symptoms (Torralva et al., 2013). Comparing young adult patients of a specialized clinic for adult ADHD with non-patient controls revealed differences on a standardized executive function assessments for immediate memory, as well as a computerized assessment for a challenging working memory and an ecological adaptation of a working memory measure (Hotel Task) that involves problem solving and working memory in the context of an employee task scenario. Even high-functioning ADHD participants showed deficits in working memory. Torralva and colleagues conclude that ADHD deficits may be observable in tasks that are ecologically valid and with tasks that have a high demand on working memory.

The current experiment was conducted in order to further attempt to observe and document potential cognitive deficits associated with ADHD, but specifically with a college student sample, which could be considered high-functioning, given their admittance $t$ to a highly selective private liberal arts college. Although such a scientific endeavor may result in no ADHD effects due to the limited demographic, finding differences at this level of academics would be highly indicative of working memory issues being prevalent in the larger population, with more diversity. Behavioral evidence indicates that college students with ADHD report more problems with attentiveness and more academic complaints, such as having to read material over and over to understand it or having trouble finishing time tests, (Lewandowski, Lovett, Codding, \& Gordon, 2008). ADHD College students have also been found to have lower high school and college GPA and withdraw from classes more than students without ADHD (Advokat, Lane, \& Luo, 2011), indicating the need to better understand the abilities of this population.

A secondary goal of this study was to use working memory capacity assessments that have been shown to correlate with fluid reasoning measures (Kane et al., 2004). The complex span measures have been widely used as assessments of working memory capacity and entail simultaneous process and memory updating (Conway et al., 2005). These measures correlated with other indicators of selective attention, and thus should be sensitive to differences based on reports of inattentiveness or ADHD diagnoses. Thus, ADHD students were compared with students with no diagnosis on tests of visual and verbal working memory, similar to the Kofler et al. (2010) study with children. Additionally, the Brown ADD scale for adults was administered to assess self-reported distractibility. The central hypothesis was that students with and without ADHD will differ in working memory performance. There was a possibility that such differences will be sensitive to memory load, which was found with children, but not yet with adults. It was also expected that students with ADHD will report more problems with attention on the Brown scale.

\section{Method}

\section{Participants}

Participants with and without a diagnosis of ADHD were recruited from the Kenyon College student population over 2 semesters (i.e., Fall, 2012 and Spring, 2013). Students were informed about the study through campus emails and public fliers that were placed in the campus bookstore and the Student Health Center, indicating that students both with and without a pre-existing diagnosis of ADHD by a physician were eligible. Since the percentage of students with a diagnosis is typically low at private colleges, the purpose of the Health Center recruitment advertisement was to attempt to reach those with ADHD to increase the likelihood of participation and thus the size of the ADHD sample for comparison. The Students volunteered in exchange for research credit in psychology courses.

\section{Materials}

Subsequent to reading and signing an informed consent form, participants completed a short demographic survey requesting information such as age, gender, and mental health history regarding attention deficit disorders. The only reported disorder for this sample was specifically Attention Deficit Hyperactivity Disorder (ADHD). Participants were advised verbally and via consent form that providing any or all of the requested information for either survey was completely voluntary and that items and measures could be skipped.

Reading Span Task. In order to assess performance for verbal working memory capacity, the Reading Span task was used, (Kane, et al., 2004). For this version of the Reading Span task, participants were instructed to read aloud a sentence displayed on the computer screen and determine whether or not it made sense with respect to the semantic content by verbalizing "Yes" or "No". At the end of each sentence there was a single capitalized letter for the participant to memorize. Participants were 
advised to take as long as needed to determine if there were semantic problems with a sentence. Once the yes/no decision was declared, the participant was informed to immediately read the letter at presented at the end of the sentence and attempt to memorize that letter for recall. Once the letter was read aloud the next sentence-letter pair was presented on the computer screen. After several sentence/letter pairs, the participant was asked to recall all the letters displayed and write them in the order of presentation. Memory set sizes for this assessment ranged from 2 to 5 sentence-letter pairs, with participants having no a priori knowledge of how many would be in any given set they were working on. There were 3 of each memory set size $(2,3,4,5)$, making a total of 42 sentence-letter pairs in the actual test. The Reading Span task began with three practice sets of sentence/letter pairs (with memory set size of 2). This particular version of the Reading Span task has good reliability (with Chronbach's alpha $=.78$ ), and construct validity (Kane et al., 2004).

Symmetry Span Task. In order to assess visual working memory capacity performance, the Symmetry Span task was used, in which both the processing and memory component were visual in nature. For the initial processing component of the task, participants viewed a pattern of shaded and nonshaded boxes on an $8 \times 8$ grid, and determined whether or not the pattern was symmetrical, with respect to a vertical axis by responding "yes" or "no". Following a verbal response in reference to vertical symmetry (and a 400 millisecond delay), the participant was briefly shown a $4 \times 4$ grid with one square shaded red (for 650 milliseconds) and asked to remember the location of the red square. After several iterations of the symmetry decisions paired with the memory grid, the participant indicated the locations of the red squares corresponding to the order displayed on a response sheet that consisted of $4 \times 4$ grids, in which the square remembered to be red was marked. A practice session involved 3 sets of 2 grid pairs, after which the testing portion presented to the participant 42 grid pairs in groups of 2 to 5 . As with the Reading Span measure, participants were not aware of the memory set sizes prior to presentation. The Symmetry Span task has also been have good reliability (with Chronbach's alpha $=.86)($ Kane et al., 2004)). Scoring for the both the Reading Span task and Symmetry Span tasks entailed calculating a total score by adding the total number of correct responses across the entire working memory task, and average total accuracy was calculated for each memory se, as well.

Brown ADD Scale. This survey requires the respondent to rate how often the quality or behavior in question applies to him/her using numbers 0 through 3; 0 corresponds to never, 1 to once per week or less, 2 to twice per week, and 3 indicating an almost daily occurrence. There are 40 items, and the total score includes adding the ratings for each. Example items are, "Listens and tries to pay attention (e.g., in a meeting, lecture, or conversation) but mind often drifts; misses out on desired information", and "Cannot complete tasks in the allotted time; needs extra time to finish satisfactorily".

\section{Procedure}

All participants were tested individually in a sound attenuated laboratory setting at the Kenyon College Cognition Lab. Each participant completed the demographic survey, and subsequently the self-report attention scale, followed by the working memory capacity assessments, with the Reading Span task administered first, followed by the Symmetry Span task. At the end of the study, participants were debriefed and provided with local information for health and counseling centers wherein they could receive assistance if concerned about attention and related cognitive functioning.

\section{Results}

Eighty-two students between the ages of 18 and 23 volunteered for the study which took place between Sept 2012 and April 2013. Mean age was 19.46 years old $(S D=1.21)$. Students with reported ADHD diagnoses comprised $9.8 \%$ of the sample. Descriptive data (means and standard error) for both working memory capacity assessments are listed in Table $\mathbf{1}$ and includes accuracy by set size for participants with and without ADHD. Total sample data, combing groups, is also included. Set size represents the amount of information to be remembered, and the means are

Reading Span Results. In order to address the hypothesis that ADHD students might show differential performance on the Reading Span task, a General Linear Model (GLM) with repeated measures was used to test the $2 \times 4$ mixed factorial design with participant group having two levels (ADHD vs. No Diagnosis) and set size with 4 levels (for memory sets of 2, 3, 4, and 5). The dependent variable is the average accuracy for each set size. Results indicated that there was a main effect of participant group on Reading Span accuracy, $(F(1,80)=4.926, p$ $=.029)$, with the ADHD group yielding lower accuracy, overall. There was no significant interaction between participant group and memory set size on accuracy, $((F(3,79)=1.142, p=.333)$. However, there was a significant main effect of memory set size on accuracy, $(F(3,79)=6.017, p=.001)$, confirming increasing task difficulty with larger memory load. Refer to Figure 1 for data for working memory performance by set size and participant group. Note that significant group differences based on ADHD diagnosis appear with the set size of 3 items, $(t(80)=$ $2.209, p=.030$, and also with set size of 4 items, $(t(80)=.664$, $p=.047)$.

Symmetry Span Results. To examine group effects on the visual working memory capacity assessment, a GLM ANOVA with repeated measures did not result in a significant main effect of ADHD diagnosis and overall performance on the Symmetry Span Task, $(F(1,80)=.744, p=.391)$. Additionally, there was no significant interaction between participant group and memory set size on average total accuracy, $(F(3,79)=$

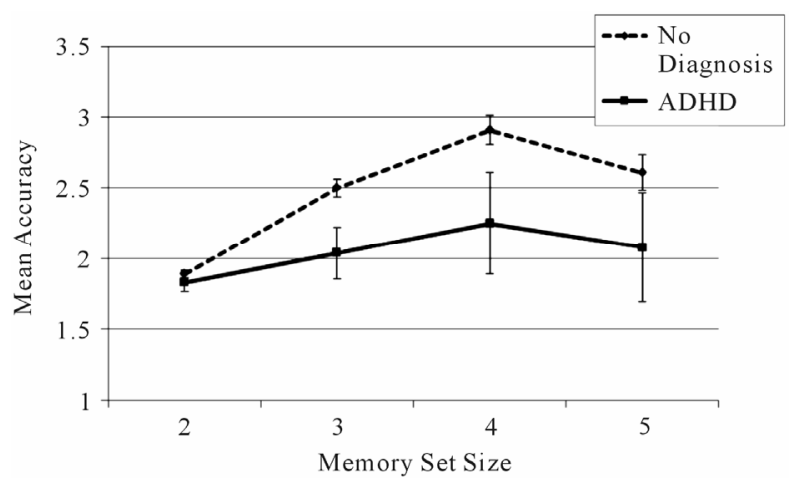

Figure 1.

Reading span memory set accuracy for participants with and without ADHD (with Standard Error Bars). 
Table 1.

Descriptive data for verbal and visual working memory measures by memory set size (Means and Standard Error).

\begin{tabular}{|c|c|c|c|c|c|c|c|c|c|}
\hline & & \multicolumn{4}{|c|}{ Verbal WM } & \multicolumn{4}{|c|}{ Visual WM } \\
\hline & & \multicolumn{4}{|c|}{ Set Size } & \multicolumn{4}{|c|}{ Set Size } \\
\hline & & 2 & 3 & 4 & 5 & 2 & 3 & 4 & 5 \\
\hline \multirow{2}{*}{ Total } & $M$ & 1.88 & 2.45 & 2.85 & 2.56 & 1.41 & 1.63 & 2.05 & 1.34 \\
\hline & $S E$ & 0.026 & 0.062 & 0.1 & 0.122 & 0.058 & 0.079 & 0.104 & 0.119 \\
\hline \multirow{2}{*}{ NonADHD } & $M$ & 1.89 & 2.5 & 2.91 & 2.61 & 1.42 & 1.63 & 2.06 & 1.4 \\
\hline & $S E$ & 0.028 & 0.065 & 0.101 & 0.128 & 0.061 & 0.085 & 0.115 & 0.127 \\
\hline \multirow{2}{*}{ ADHD } & $M$ & 1.83 & 2.04 & 2.25 & 2.08 & 1.25 & 1.63 & 1.96 & 0.79 \\
\hline & $S E$ & 0.063 & 0.183 & 0.361 & 0.387 & 0.197 & 0.213 & 0.117 & 0.281 \\
\hline
\end{tabular}

$1.529, p=.208)$. As with the Reading Span task, there was also a significant main effect of set size on accuracy, $(F(3,79)=$ $12.66, p<.001)$. Additionally, total accuracy on the visual and verbal working memory tasks did significantly correlate with each other $(r=.342, p=.002)$, and each task yielded fairly good reliability for this sample, with Chronbach's alpha at .69 for the Reading Span Task and .84 for Symmetry Span.

Test Modality and Set Size. A GLM ANOVA was used to examine effects and interactions between test modality (verbal, visual), memory set size $(2,3,4,5)$, as within-subject factors and ADHD status as the between-subjects variable. Mean accuracy was the dependent variable in this analysis. Results revealed a significant main effect of test modality, $(F(1,81)=$ $32.69, p<.001)$, with Reading Span having higher overall accuracy over Symmetry Span. There was also a significant main effect of memory set size, $(F(3,79)=12.812, p<.001)$, as well as a significant interaction between set size and test modality, $(F(3,79)=4.446, p=.005)$. Refer to Figure 2 for the modality by set size interaction on accuracy. Note the differences in the working memory tasks, with verbal yielding higher memory accuracy, but both tasks showing performance increase with set size until the set size with 5 items is reached. There is a significant drop in performance for all participants from set size 4 to 5 on the Reading Span task, $(t(81)=2.561, p=.012)$, and also for the Symmetry Span task, $(t(81)=7.095, p<.001)$.

Test Modality, Set Size, and ADHD. There was no significant interaction between test modality and ADHD group status, $(F(1,81)=.600, p=.441)$, nor was there a significant interaction between set size and participant groups, $(F(3,79)=1.263$, $p=.288)$. Additionally, there was no interaction between modality, set size, and participant group, $(F(3,79)=1.351, p$ $=.259$ ). Lastly, there was no main effect of ADHD status on overall working memory performance (which combines both modalities $),(F(1,81)=3.118, p=.081)$, however it appears to be approaching significance.

Brown Scale. The mean for the totaled ratings was 41.768 , $(S D=16.866)$, with the range of scores from 8 to 95 , with a higher score indicating more reported distraction and problems with attention. Results of an independent samples t-test indicated no significant difference between ADHD participants and those without a diagnosis, $(t(80)=-1.926, p=.058)$, however, there was a trend with ADHD students having a higher total score, which indicates a higher distraction ratings, $\left(M_{\text {NonADHD }}=\right.$ $40.608, S D_{N o n A D H D}=15.971$ and $M_{A D H D}=52.500, S D_{A D H D}=$

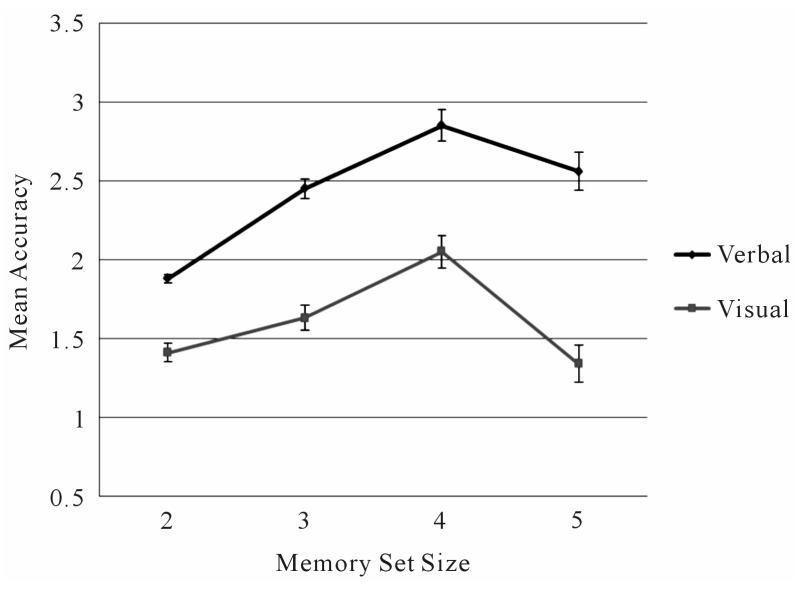

Figure 2.

Mean accuracy on verbal and visual working measures as a function of memory set size (with Standard Error Bars).

22.045). The Brown Scale did not significantly correlate with Reading Span $(r=-.60, p=.591)$, or Symmetry Span $(r=.00$, $p=.997)$.

\section{Discussion and Conclusion}

The important finding of this study was that the college undergraduates with an ADHD diagnosis did show difficulty on at least one of the working memory measures, the Reading Span task. Since the working memory system is comprised of domain-specific processing with a central executive component for controlled attention, it is necessary to examine memory load effects by comparing performance with increasing set size. Changes in performance as set size increases are an indicator of whether the differences between groups is based on the central executive component or on the addition of constraints on amount of information for verbal rehearsal. By closely examining the Reading Span data (Figure 1), it is important to note that there is no change in the performance accuracy of the ADHD group as set size increases. This group can recall about 2 items, in general, regardless of set size. Participants without a diagnosis show a significant increase in recall along with memory load. The two groups clearly differ in performance patterns up to set size of 4 , followed by a drop in performance for those 
without ADHD to match the accuracy of the ADHD students. This change from set size 4 to 5 indicates a capacity limitation and overload for the non-diagnosed group, whereas the ADHD individuals remain the same, with maximum recall at 2 . The data pattern is consistent with the notion that ADHD students have limitations on verbal rehearsal capacity that are related to the deficits in attention and sustained effort. The results also contribute to the concurrent validity of the Reading Span task as a complex span that is measure sensitive to individual differences in the ability to control attention (Engle, 2002), since there is now evidence of its sensitivity to differences in ADHD diagnosis.

The visual complex span task appeared to be significantly more difficult than the verbal assessment for working memory capacity. As seen in Figure 2, performance is lower for all participants on the Symmetry Span task, which could explain the lack of group differences in recall accuracy. Both groups perform similarly on the memory sets for Symmetry Span, with separations in performance emerging with set size of 5, but still not a significant difference (refer to Table 1 for means). This task may be too challenging to bring out differences in ADHD status. Additionally, this test came at the end of the battery of assessments and could be affected by mental fatigue.

The Brown scale scores for reported inattentiveness were not different for the participant groups, although the difference was approach statistical significance $(p=.058)$, with the ADHD group reporting more problems with attention. The Brown scale is widely used as an indicator of ADHD symptoms, however, it is only one assessment that is often used in combination with other measures for the purpose of diagnosis. Previous research has not always found a relationship with this scale and working memory measures (Stearns, Dunham, McIntosh, \& Dean, 2004), and it is possible that cognitive performance measures may be more accurate indicators of ADHD than self-reported behavior.

The main limitation of the research is the small sample size of the ADHD group (9.8\%), which is perhaps unavoidable when studying college populations in which admittance is dependent upon meeting high academic entry criteria. However, the findings of this study are theoretically consistent and are similar to findings with children (Kofler et al., 2010). Revealing individual differences in cognitive constraints is highly important to understanding the intellectual capacity of college students in the academic setting, the authors suggest that researchers continue to investigate the relationship between working memory and ADHD at other institutions for comparison to the results of this study.

The findings of this research provide converging evidence with previous research with young adult patients and college students with ADHD indicating lower performance on a variety of working memory assessments in comparison to those without a diagnosis (Roberts, Millich, \& Fillmore, 2012; Torralva et al., 2013). To put these findings in context, the working memory measures assess the ability to maintain information in consciousness while alternately engaged in another cognitive activity. In the reading span task, reading and comprehension judgments happen while trying to additively remember a list of letters. Memory updating and attempted rehearsal of the letters occur while one is "distracted" by the reading aspect. Having this extra cognitive processing reduces the amount of information that can be maintained in comparison to having no distracting activity, regardless of a diagnosis. The findings reveal that students with ADHD hold less in memory when engaged in this dual-task context. The results have implications for situations in which college students with ADHD are under cognitive load. Knowledge that problems with working memory in ADHD individuals extend into college should be integrated with findings from research on methods to compensate for potential difficulties and understanding factors involved in overcoming obstacles for these students.

\section{REFERENCES}

Advokat, C., Lane, S. M., \& Luo, C. (2011). College students with and without ADHD: Comparison of self-report of medication usage, study habits, and academic achievement. Journal of Attention Disorders, 15, 656-666. doi:10.1177/1087054710371168

Bleckley, M. K., Durso, F. T., Crutchfield, J. M., Engle, R. W., \& Khanna, M. M. (2003). Individual differences in working memory capacity predict visual attention allocation. Psychonomic Bulletin \& Review, 10, 884-889. doi:10.3758/BF03196548

Caterino, L. C., \& Verdi, M. P. (2012). Is the recall of verbal-spatial information from working memory affected by symptoms of ADHD? Journal of Attention Disorders, 16, 562-571. doi: $10.1177 / 1087054711403713$

Conway, A. R. A., Cowan, N., \& Bunting, M. F. (2001). The cocktail party phenomenon revisited: The importance of working memory capacity. Psychonomic Bulletin and Review, 97, 332-361. doi:10.3758/BF03196169

Conway, A. R. A., Kane, M. J., Bunting, M. F., Hambrick, D. Z., Wilhelm, O., \& Engle, R. W. (2005). Working memory span tasks: A methodological review and user's guide. Psychonomic Bulletin and Review, 12, 769-786. doi:10.3758/BF03196772

Daneman, M., \& Carpenter, P. A. (1980). Individual differences in working memory and reading. Journal of Verbal Learning \& Verbal Behavior, 19, 450-466. doi:10.1016/S0022-5371(80)90312-6

Engle, R. W. (2002).Working memory capacity as executive attention. Current Directions in Psychological Science, 11, 19-23. doi: $10.1111 / 1467-8721.00160$

Kane, M. J., Hambrick, D. Z., Tuholski, S. W., Wilhelm, O., Payne, T. W., \& Engle, R. W. (2004). The generality of working memory capacity: A latent-variable approach to verbal and visuospatial memory span and reasoning. Journal of Experimental Psychology: General, 133, 189-217. doi:10.1037/0096-3445.133.2.189

Kofler, M. J., Rapport, M. D., Bolden, J., Sarver, D. E., \& Raiker, J. S. (2010). ADHD and working memory: The impact of central executive deficits and exceeding storage/rehearsal capacity on observed inattentive behavior. Journal of Abnormal Child Psychology, 38, 149161. doi:10.1007/s10802-009-9357-6

Lewandowski, L. J., Lovett, B. J., Codding, R. S., \& Gordon, M. (2008). Symptoms of ADHD and academic concerns in college students with and without ADHD diagnoses. Journal of Attention Disorders, 12, 156161. doi: $10.1177 / 1087054707310882$

Payne, T. W., Kalibatseva, Z., \& Jungers, M. (2009). Does domain experience compensate for working memory capacity when predicting second language reading comprehension? Learning and Individual Differences, 19, 119-123. doi:10.1016/j.lindif.2008.05.003

Roberts, W., Milich, R., \& Fillmore, M. T. (2012). Constraints on in formation processing capacity in adults with ADHD. Neuropsychology, 26, 695-703. doi:10.1037/a0030296

Stearns, C., Dunham, M., McIntosh, D, \& Dean, R.S. (2004). Attention deficit/hyperactivity disorder and working memory in clinically referred adults. International Journal of Neuroscience, 114, 273-287. doi:10.1080/00207450490269499

Torralva, T., Gleichgerrcht, E., Lischinsky, A., Roca, M., \& Manes, F. (2013). "Ecological" and highly demanding executive tasks detect real-life deficits in high-functioning adult ADHD patients. Journal of Attention Disorders, 17, 11-19. doi:10.1177/1087054710389988

Unsworth, N., \& Engle, R. W. (2005). Individual differences in working memory capacity and learning: Evidence from the serial reaction time task. Memory \& Cognition, 33, 213-220. doi:10.3758/BF03195310 